\title{
Is CA72-4 a Useful Biomarker in Differential Diagnosis between Ovarian Endometrioma and Epithelial Ovarian Cancer?
}

\author{
Emanuela Anastasi, ${ }^{1}$ Lucia Manganaro, ${ }^{2}$ Teresa Granato, ${ }^{3}$ Pierluigi Benedetti Panici, ${ }^{4}$ \\ Luigi Frati, ${ }^{1}$ and Maria Grazia Porpora ${ }^{4}$ \\ ${ }^{1}$ Department of Molecular Medicine, "Sapienza” University of Rome, Policlinico Umberto I, Viale Regina Elena 324, \\ 00161 Rome, Italy \\ ${ }^{2}$ Department of Radiological, Oncological and Pathological Sciences, "Sapienza" University of Rome, Policlinico Umberto I, \\ Viale Regina Elena 324, 00161 Rome, Italy \\ ${ }^{3}$ CNR-IBPM, National Research Council, Viale Regina Elena 324, 00161 Rome, Italy \\ ${ }^{4}$ Department of Gynecology, Obstetrics and Urology, "Sapienza" University of Rome, Policlinico Umberto I, Viale Regina Elena 324, \\ 00161 Rome, Italy
}

Correspondence should be addressed to Emanuela Anastasi; emanuela.anastasi@uniroma1.it

Received 26 June 2013; Accepted 8 September 2013

Academic Editor: Alex J. Rai

Copyright (C) 2013 Emanuela Anastasi et al. This is an open access article distributed under the Creative Commons Attribution License, which permits unrestricted use, distribution, and reproduction in any medium, provided the original work is properly cited.

Background. Surgical excision of ovarian endometriomas in patients desiring pregnancy has recently been criticized because of the risk of damage to healthy ovarian tissue and consequent reduction of ovarian reserve. A correct diagnosis in cases not scheduled for surgery is therefore mandatory in order to avoid unexpected ovarian cancer misdiagnosis. Endometriosis is often associated with high levels of CA125. This marker is therefore not useful for discriminating ovarian endometrioma from ovarian malignancy. The aim of this study was to establish if the serum marker CA72-4 could be helpful in the differential diagnosis between ovarian endometriosis and epithelial ovarian cancer. Methods. Serums CA125 and CA72-4 were measured in 72 patients with ovarian endometriomas and 55 patients with ovarian cancer. Results. High CA125 concentrations were observed in patients with ovarian endometriosis and in those with ovarian cancer. A marked difference in CA72-4 values was observed between women with ovarian cancer $(71.0 \%)$ and patients with endometriosis $(13.8 \%)(P<0.0001)$. Conclusions. This study suggests that CA72-4 determination can be useful to confirm the benign nature of ovarian endometriomas in women with high CA125 levels.

\section{Introduction}

Endometriosis is a common chronic disease, affecting $5-10 \%$ of women in reproductive age [1]. The disease is characterized by the presence and growth of endometrial tissue outside the uterine cavity, often associated with infertility and pelvic pain and that tends to recur [2-5]. Endometriosis can be diagnosed by clinical and ultrasound examinations (US), but the most accurate procedure to confirm the diagnosis is laparoscopy that allows visualization of lesions and histological confirmation [6].

Endometriosis is a benign disease but it shares several characteristics with invasive cancer. Cancer antigen 125 (CA125) is a tumor marker used for the differential diagnosis in a postmenopausal woman with an adnexal mass [7]. However, in premenopausal age, CA125 is characterized by a low diagnostic specificity, as abnormally high concentrations can be found in malignancies of different origin including nonovarian gynecological cancer [8], in women with nongynecological diseases such as tuberculosis and liver cirrhosis, and also in pelvic inflammatory disease, uterine fibroids, or physiological conditions such as pregnancy or different phases of the menstrual cycle $[9,10]$. In patients with endometriosis, CA125 levels can be high. In fact, CA125 is the most extensively investigated and used peripheral biomarker for monitoring the disease [11]. Thus, CA125 has a limited role in the differential diagnosis between endometriosis and ovarian cancer due to the lack of specificity [12]. Surgical excision 
of ovarian endometriomas in patients desiring pregnancy has recently been criticized because of the risk of damage to healthy ovarian tissue and consequent reduction of ovarian reserve $[6,13,14]$. In cases unscheduled for surgery, particularly in women undergoing assisted reproductive techniques, it is mandatory to rule out an ovarian malignancy before ovarian stimulation and embryo-transfer [15]. Misdiagnosed ovarian cancer has been found in women with suspected ovarian endometriosis [16, 17].

Therefore identification of noninvasive and accessible markers of epithelial ovarian carcinoma (EOC) is valuable. For this reason serum tumor markers are being increasingly used for the differential diagnosis of adnexal masses.

Among these, cancer antigen 72-4 (CA72-4), a glycoprotein, which increases in gastric, colon, breast, and ovarian adenocarcinomas, may be employed alone or in combination with CA125. CA72-4 is less sensitive than CA125 for EOC, but it is not influenced by pregnancy or the menstrual cycle, and it is only slightly influenced by inflammatory conditions $[18,19]$.

The aim of this study was to evaluate the role of CA72-4 in the differential diagnosis between ovarian cancer and ovarian cystic endometriosis.

\section{Patients and Methods}

From June 2012 to February 2013, 127 consecutive Italian women (mean age: 50 years, range: $24-74$ ) referred to the Department of Gynecology, Obstetrics and Urology at the University of Rome "Sapienza" for the presence of an adnexal mass, detected at clinical and ultrasound (US) examinations, were enrolled in the study.

Exclusion criteria included current hormonal therapy, pregnancy, chronic diseases, or other types of cancer. All patients signed written informed consent. At enrolment, medical history was collected and peripheral blood samples were drawn from all women and immediately sent to the laboratory for analysis of tumor markers. All groups underwent complete physical examination and abdominal and transvaginal US.

The women were divided into the following 2 groups.

Group A. It consisted of 72 patients with ovarian endometrioma (mean age: 36 years, range: $24-48$ ). Diagnosis of endometriosis was achieved on the basis of medical history and clinical and pelvic transabdominal and/or transvaginal US examinations. Patients with indeterminate findings underwent pelvic magnetic resonance imaging (MRI) to confirm suspected endometriosis using the previously described technique $[20,21]$. At laparoscopy, all endometriomas and lesions were excised, and the disease was staged according to the rASRM classification [22]. Mean diameter of endometriomas was $33 \pm 18.9 \mathrm{~mm}$ (range 10-80). Histological examination confirmed the diagnosis in all cases.

Group B. It consisted of 55 patients with ovarian carcinoma (EOC) (mean age: 65 years, range: 40-74). All women
TABLE 1: Patient population characteristics.

\begin{tabular}{lcccccc}
\hline \multirow{2}{*}{ Diagnosis } & \multirow{2}{*}{ Mean age (years) } & $n$ & \multicolumn{4}{c}{ Classification } \\
& & & I & II & III & IV \\
\hline & 36 & 72 & - & 7 & 30 & 35 \\
Group A (endometriosis) & 36 & \multicolumn{5}{c}{ FIGO stage } \\
\hline & 65 & 55 & 5 & 4 & 10 & 36 \\
\hline Group B (EOC) & & & & &
\end{tabular}

underwent surgery. Staging was made according to the International Federation of Gynecology and Obstetrics (FIGO) [23]. Histology confirmed the diagnosis in all cases.

Patient characteristics are summarized in Table 1.

2.1. Sample Preparation. All sera were acquired following a standard collection protocol. Briefly, samples were collected in a Red Top Vacutainer, clotted 60-90 min, and centrifuged for $10 \mathrm{~min}$ at $1300 \times \mathrm{g}$. The serum fractions were aliquoted and stored at $-80^{\circ} \mathrm{C}$ until analysis.

2.2. CA125 Assay. Lumipulse G1200 CA125II is an assay system for the quantitative measurement of CA125 in specimens based on chemiluminescent enzyme immunoassay technology (CLEIA) by a two-step sandwich method (InnogeneticsFujirebio, Belgium; Japan). This assay makes use of solid phase and ALP-labeled monoclonal antibodies (OC125 and M11, resp.).

CA125 in specimens specifically binds to anti-CA125 monoclonal antibody immobilized on the particles forming antigen-antibody immunocomplexes. The particles are then washed and rinsed in order to remove unbound materials. Alkaline phosphatase (ALP)-labeled anti-CA125 monoclonal antibody specifically binds to CA125 of the immunocomplexes. After a second wash, substrate solution is added. AMPPD contained in the substrate solution is dephosphorylated by the catalysis of ALP indirectly conjugated to the particles. A luminescent signal is generated by the cleavage reaction of dephosphorylated 3-( $2^{\prime}$-spiroadamantyl)-4-methoxy4 -( $3^{\prime \prime}$-phosphoryloxy)-phenyl-1,2-dioxetane (AMPPD) and reflects the amount of CA125 in the sample. Normal levels of CA125 were $<35 \mathrm{U} / \mathrm{mL}$.

2.3. CA72-4 Assay. CA72-4 was detected utilizing a solid phase two-site immunoradiometric ELSA-CA72-4 assay (Cisbio Bioassays, France). Two monoclonal antibodies were prepared against sterically remote antigenic sites on the TAG 72 molecule: the first was coated on the ELSA solid phase; the second, radiolabeled with iodine 125, was used as tracer. TAG 72 molecules present in the standards or the samples to be tested were "sandwiched" between the two antibodies. Following the formation of the coated antibody/antigen/antibody sandwich, the unbound 7 tracer was easily removed by a washing step. The radioactivity bound to the ELSA was proportional to the concentration of TAG 72 present in the sample. Normal levels of CA72-4 were considered to be $<3.8 \mathrm{U} / \mathrm{mL}$. 


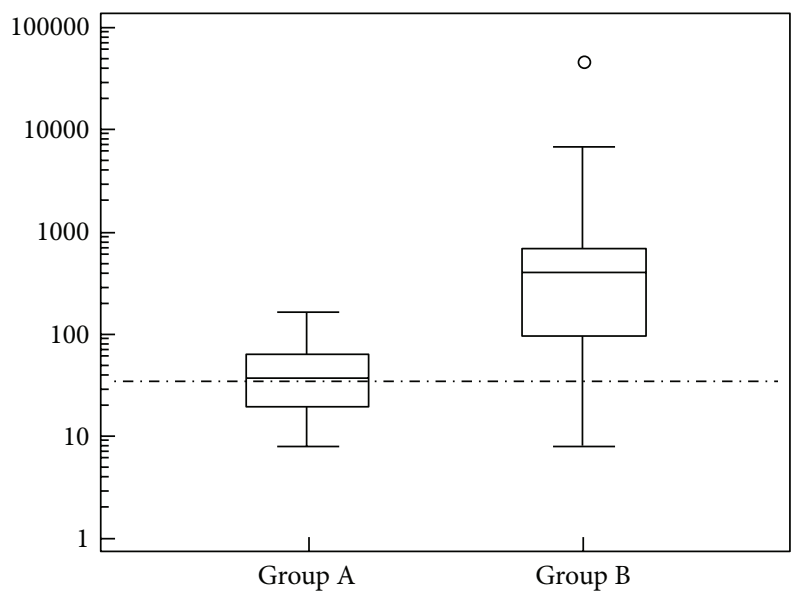

(a)

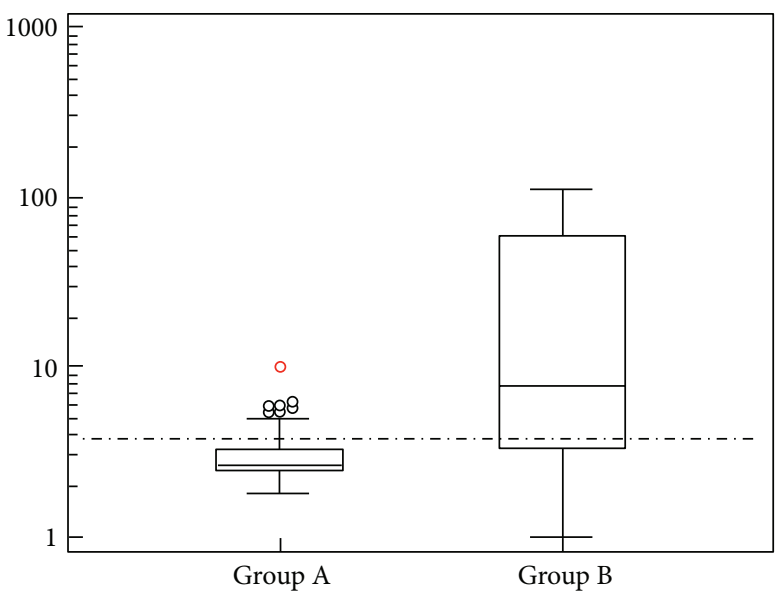

(b)

Figure 1: (a) Box and whisker plots representing median levels and the interquartile range (box) of CA125 for each group. The dashed horizontal line represents the cut-off level for CA125 $(35 \mathrm{U} / \mathrm{mL})$. The $y$-axis is a logarithmic scale. Group A=endometriosis; Group $\mathrm{B}=$ epithelial ovarian cancer. (b) Box and whisker plots representing median levels and the interquartile range (box) of CA72-4 for each studied group. The dashed horizontal line represents the cut-off level for CA72-4 $(3.8 \mathrm{U} / \mathrm{mL})$. The $y$-axis is a logarithmic scale. Group $\mathrm{A}=$ endometriosis; Group B = epithelial ovarian cancer.

2.4. Statistical Analysis. Women were stratified by disease in two groups. In each group, the median, range, mean, and SD for serum CA125 and CA72-4 levels were determined. MannWhitney test was used to assess the difference in distributions of tumor markers between different patient populations. Log base 10-transformed whisker-box plots were generated for each marker by disease group. Receiver operator characteristic (ROC) curves were constructed, and the areas under the curve (AUC) with binomial exact 95\% confidence intervals (95\% CI) were calculated. The method described by DeLong et al. was used to calculate the difference between two AUCs. For all statistical comparisons, a level of $P<0.05$ was accepted as statistically significant. All statistical analyses were performed using MedCalc v.12.2.1.0.

\section{Results}

3.1. Biomarker Distribution. CA125 and CA72-4 serum marker levels were evaluated in all groups (127 women). Results expressed as median and ranges are shown in Table 2.

In group A, CA125 was high in 54.1\% and CA72-4 was slightly increased in only $13.8 \%(10 / 72)$ of the cases; this increase was not statistically significant (Figure 1(a)).

In group B, CA125 above the normal value was observed in $89.1 \%$ of the patients, whereas CA72- 4 was increased in $71 \%$ of cases (Figure $1(\mathrm{~b})$ ).

A statistically significant difference was found between CA72-4 levels in group B versus group A $(P<0.0001)$ (Table 2).

3.2. Diagnostic Accuracy. Diagnostic performance of the markers in discriminating malignant from benign gynecologic conditions was verified using ROC analysis. The two markers showed good performance, with AUCs of 0.86 and 0.81 for CA125 and CA72-4, respectively.
TABLE 2: Statistical characteristics of serum markers for each group.

\begin{tabular}{lccc}
\hline & & $\begin{array}{c}\text { Group A } \\
\text { endometriosis }\end{array}$ & $\begin{array}{c}\text { Group B } \\
\text { EOC }\end{array}$ \\
\hline \multirow{2}{*}{ CA125 } & Mean & 72 & 55 \\
$\mathrm{U} / \mathrm{mL}$ & $\mathrm{SD}$ & 45.0 & 1976.3 \\
& median (range) & $37(8-167)$ & 7390.4 \\
\hline \multirow{2}{*}{$\mathrm{CA72-4}$} & Mean & 3.1 & 32.55 \\
$\mathrm{U} / \mathrm{mL}$ & $\mathrm{SD}$ & 1.28 & 40.2 \\
& median (range) & $2.7(1.8-10)$ & $8(1-112)^{\mathrm{a}}$ \\
\hline
\end{tabular}

${ }^{\mathrm{a} P}$ value $<0.0001$. Group B versus Group A.

\section{Discussion}

Endometriosis is a known cause of CA125 elevation and represents a common gynecologic disorder in women of reproductive age. Generally the diagnosis of ovarian endometriosis is made by clinical and imaging technique examinations [24] and confirmed by surgery with histological examination [6]. Recently surgical treatment of ovarian endometriosis in women desiring pregnancy has been criticized because of the risk of ovarian healthy tissue damage $[13,14]$. Therefore, in selected cases with ovarian endometrioma treated by medical therapy or undergoing assisted reproductive techniques (ART) without prior surgery, a correct diagnosis is mandatory. In these cases, the use of tumor markers with high sensitivity and specificity could help to reduce the risk, even if small, of undetected ovarian cancer. In fact, there is a recognized association between endometriosis and clear cell, low-grade serous, and endometrioid ovarian cancer [25].

In this study, we investigated CA125 and CA72-4 in the diagnostic evaluation of ovarian endometrioma. CA125 
is frequently increased in patients with endometriosis and used for monitoring the disease. In agreement with data reported in the literature [26], more than $50 \%$ of women with endometriosis expressed high levels of CA125. Therefore in the differential diagnosis between EOC and endometrioma, CA125 is not a reliable marker yielding a sensitivity of $89.1 \%$ and specificity of only $54.1 \%$.

In our study a slight and not statistically significant increase of CA72-4 was found in a small number of patients with endometriosis, with the highest observed value of $10 \mathrm{U} / \mathrm{mL}$, which is a borderline value, found only in one woman. High levels of CA72-4 were found in $71 \%$ of patients with EOC, and the difference in CA72-4 levels between women with endometriosis and those with EOC was statistically significant. The relationship between CA72-4 levels and FIGO stage is still under evaluation. Unfortunately, since epithelial ovarian cancer is often diagnosed at late stages, most EOC patients in our study were at advanced stages of disease. Possible differences in CA72-4 values in the different stages of disease were furthermore not evaluated, as it was not an objective of our study.

In conclusion, our data confirm the results reported by Lenhard et al. who showed that CA125 but not CA72-4 tends to be increased in the presence of endometriosis [18]. Recently a new marker, HE4, has been used for its high sensitivity and specificity; however, there are some cases with benign or physiological conditions in which high levels can be found [27]. Therefore CA72-4 evaluation may have a role in the differentiation between malignant and ovarian endometriosis in selected patients.

\section{Acknowledgment}

The authors are thankful to Adele Ticino, Renato Falzarano, Giuseppina Gennarini, and Valentina Viggiani for their technical assistance.

\section{References}

[1] B. Eskenazi and M. L. Warner, "Epidemiology of endometriosis," Obstetrics and Gynecology Clinics of North America, vol. 24, no. 2, pp. 235-258, 1997.

[2] L. C. Giudice, "Endometriosis," The New England Journal of Medicine, vol. 362, no. 25, pp. 2389-2398, 2010.

[3] M. G. Porpora, P. R. Koninckx, J. Piazze, M. Natili, S. Colagrande, and E. V. Cosmi, "Correlation between endometriosis and pelvic pain," Journal of the American Association of Gynecologic Laparoscopists, vol. 6, no. 4, pp. 429-434, 1999.

[4] M. G. Porpora, D. Pallante, A. Ferro, B. Crisafi, F. Bellati, and P. Benedetti Panici, "Pain and ovarian endometrioma recurrence after laparoscopic treatment of endometriosis: a long-term prospective study," Fertility and Sterility, vol. 93, no. 3, pp. 716$721,2010$.

[5] M. E. Coccia, F. Rizzello, A. Palagiano, and G. Scarselli, "Longterm follow-up after laparoscopic treatment for endometriosis: multivariate analysis of predictive factors for recurrence of endometriotic lesions and pain," European Journal of Obstetrics Gynecology and Reproductive Biology, vol. 157, no. 1, pp. 78-83, 2011.
[6] L. J. E. W. van Dijk, W. L. D. M. Nelen, T. M. D’Hooghe et al., "The European Society of Human Reproduction and Embryology guideline for the diagnosis and treatment of endometriosis: an electronic guideline implementability appraisal," Implementation Science, vol. 6, no. 1, article 7, 2011.

[7] L. Bordin, C. Fiore, G. Don et al., "Evaluation of erythrocyte band 3 phosphotyrosine level, glutathione content, CA-125, and human epididymal secretory protein E4 as combined parameters in endometriosis," Fertility and Sterility, vol. 94, no. 5, pp. 1616-1621, 2010.

[8] J. M. Escudero, J. M. Auge, X. Filella, A. Torne, J. Pahisa, and R. Molina, "Comparison of serum human epididymis protein 4 with cancer antigen 125 as a tumor marker in patients with malignant and nonmalignant diseases," Clinical Chemistry, vol. 57, no. 11, pp. 1534-1544, 2011.

[9] R.-H. He, W.-M. Yao, L.-Y. Wu, and Y.-Y. Mao, "Highly elevated serum CA-125 levels in patients with non-malignant gynecological diseases," Archives of Gynecology and Obstetrics, vol. 283, no. 1, pp. S107-S110, 2011.

[10] M. R. McLemore, B. E. Aouizerat, K. A. Lee et al., "A comparison of the cyclic variation in serum levels of CA125 across the menstrual cycle using two commercial assays," Biological Research For Nursing, vol. 14, no. 3, pp. 250-256, 2012.

[11] S. Gupta, A. Agarwal, L. Sekhon, N. Krajcir, M. Cocuzza, and T. Falcone, "Serum and peritoneal abnormalities in endometriosis: potential use as diagnostic markers," Minerva Ginecologica, vol. 58, no. 6, pp. 527-551, 2006.

[12] R. C. Bast Jr., T. L. Klug, and E. St. John, "A radioimmunoassay using a monoclonal antibody to monitor the course of epithelial ovarian cancer," The New England Journal of Medicine, vol. 309, no. 15 , pp. $883-887,1983$.

[13] I. Tsoumpou, M. Kyrgiou, T. A. Gelbaya, and L. G. Nardo, "The effect of surgical treatment for endometrioma on in vitro fertilization outcomes: a systematic review and meta-analysis," Fertility and Sterility, vol. 92, no. 1, pp. 75-87, 2009.

[14] F. Raffi, M. Metwally, and S. Amer, "The impact of excision of ovarian endometrioma on ovarian reserve: a systematic review and meta-analysis," The Journal of Clinical Endocrinology \& Metabolism, vol. 97, no. 9, pp. 3146-3154, 2012.

[15] N. F. Vlahos, K. P. Economopoulos, and S. Fotiou, "Endometriosis, in vitro fertilisation and the risk of gynaecological malignancies, including ovarian and breast cancer," Best Practice and Research, vol. 24, no. 1, pp. 39-50, 2010.

[16] D. Zygouris, V. Leontara, G. M. Makris et al., "Endometrioid ovarian cancer arising from an endometriotic cyst in a young patient," European Journal of Gynaecological Oncology, vol. 33, pp. 324-325, 2012.

[17] T. Vasilakaki, E. Skafida, E. Arkoumani, X. Grammatoglou, N. Firfiris, and K. Manoloudaki, "Borderline clear cell adenofibroma of the ovary associated with ovarian endometriosis: a case report," European Journal of Gynaecological Oncology, vol. 33, pp. 230-232, 2012.

[18] M. S. Lenhard, S. Nehring, D. Nagel et al., "Predictive value of CA 125 and CA 72-4 in ovarian borderline tumors," Clinical Chemistry and Laboratory Medicine, vol. 47, no. 5, pp. 537-542, 2009.

[19] T. Granato, C. Midulla, F. Longo, B. Colaprisca, L. Frati, and E. Anastasi, "Role of HE4, CA72.4, and CA125 in monitoring ovarian cancer," Tumor Biology, vol. 33, no. 5, pp. 1335-1339, 2012. 
[20] L. Manganaro, F. Fierro, A. Tomei et al., "Beyond laparoscopy: 3T magnetic resonance imaging in the evaluation of posteriorculde-sac obliteration," Magnetic Resonance Imaging, vol. 30, pp. 1432-1438, 2011.

[21] L. Manganaro, F. Fierro, A. Tomei et al., "Feasibility of $3.0 \mathrm{~T}$ pelvic MR imaging in the evaluation of endometriosis," European Journal of Radiology, vol. 81, no. 6, pp. 1381-1387, 2011.

[22] American Society for Reproductive Medicine, "Revised American Society for Reproductive Medicine classification of endometriosis," Fertility and Sterility, vol. 67, no. 5, pp. 817-821, 1997.

[23] W. C. Helm, Ovarian Cancer Staging, 2011.

[24] C. Van Holsbeke, B. Van Calster, S. Guerriero et al., "Endometriomas: their ultrasound characteristics," Ultrasound in Obstetrics and Gynecology, vol. 35, no. 6, pp. 730-740, 2010.

[25] C. L. Pearce, C. Templeman, M. A. Rossing et al., "Association between endometriosis and risk of histological subtypes of ovarian cancer: a pooled analysis of case-control studies," The Lancet Oncology, vol. 13, no. 4, pp. 385-394, 2012.

[26] R. G. Moore, M. C. Miller, M. M. Steinhoff et al., "Serum HE4 levels are less frequently elevated than CA125 in women with benign gynecologic disorders," American Journal of Obstetrics and Gynecology, vol. 206, no. 4, pp. 351.el-351.e8, 2012.

[27] E. Anastasi, T. Granato, R. Falzarano et al., "The use of HE4, CA125 and CA72-4 biomarkers for differential diagnosis between ovarian endometrioma and epithelial ovarian cancer," Journal of Ovarian Research, vol. 6, pp. 1-8, 2013. 


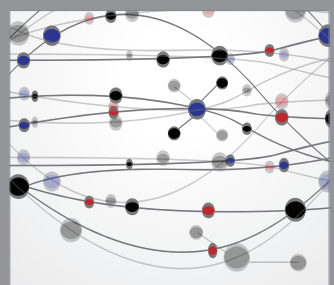

The Scientific World Journal
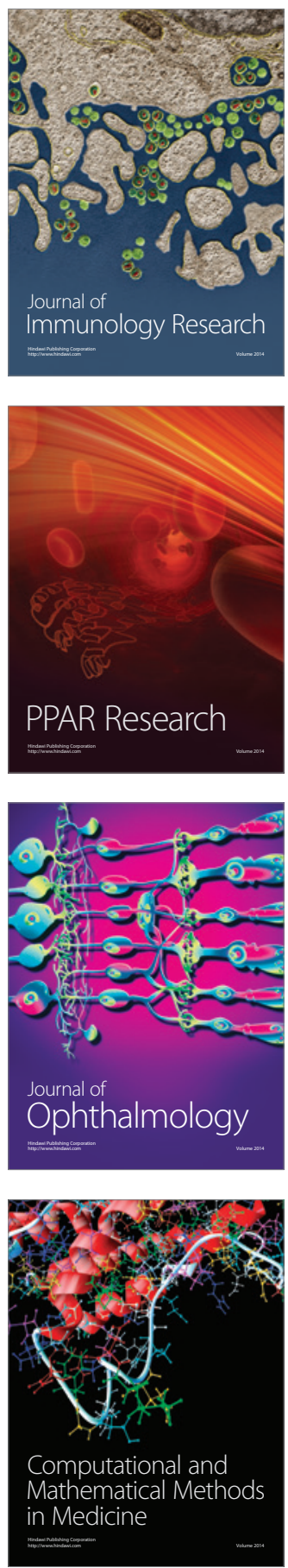

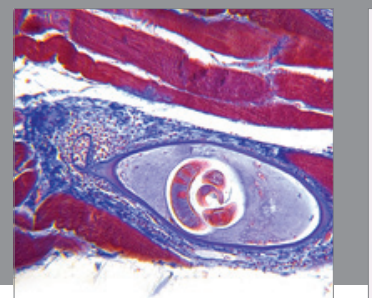

Gastroenterology

Research and Practice
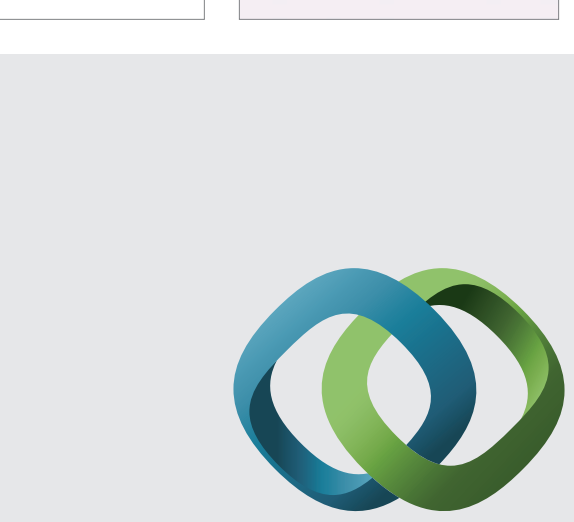

\section{Hindawi}

Submit your manuscripts at

http://www.hindawi.com
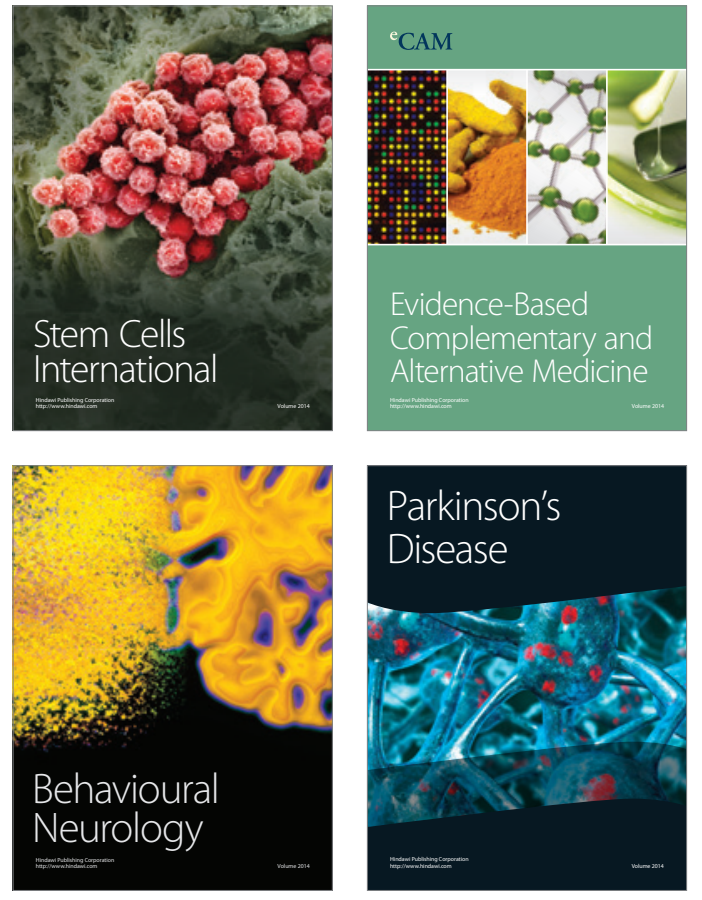
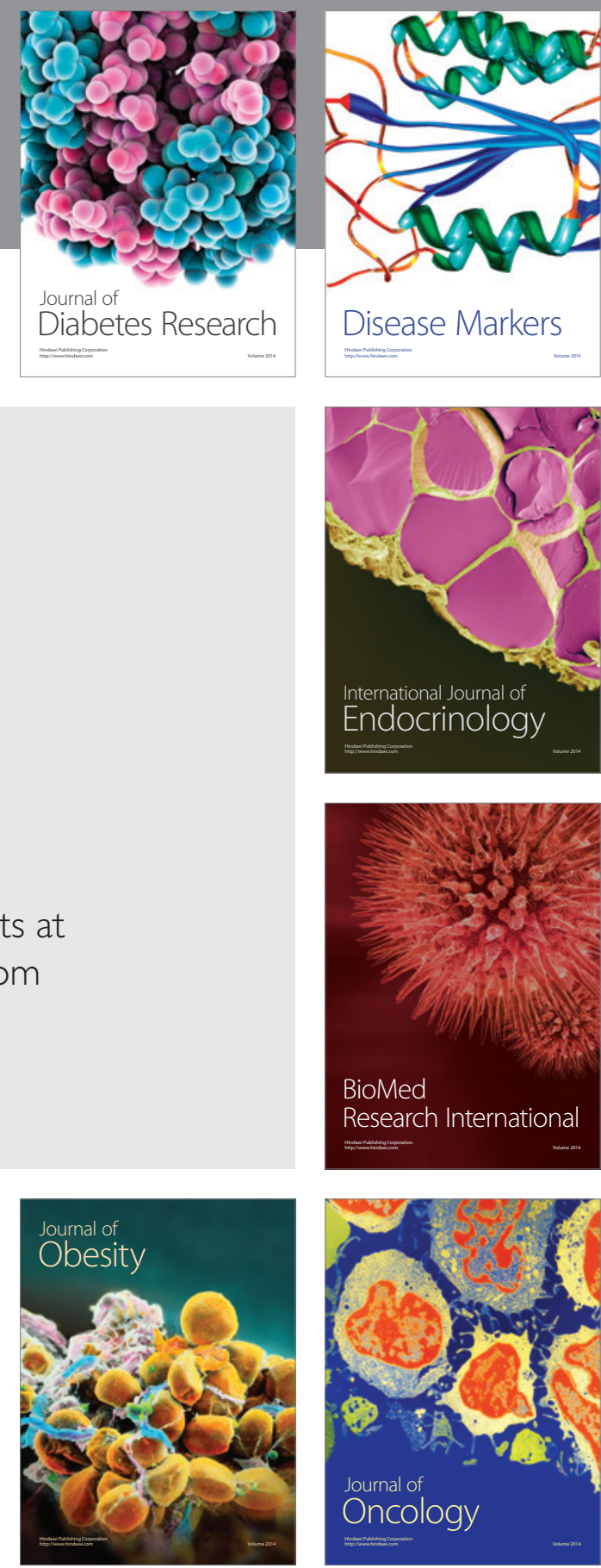

Disease Markers
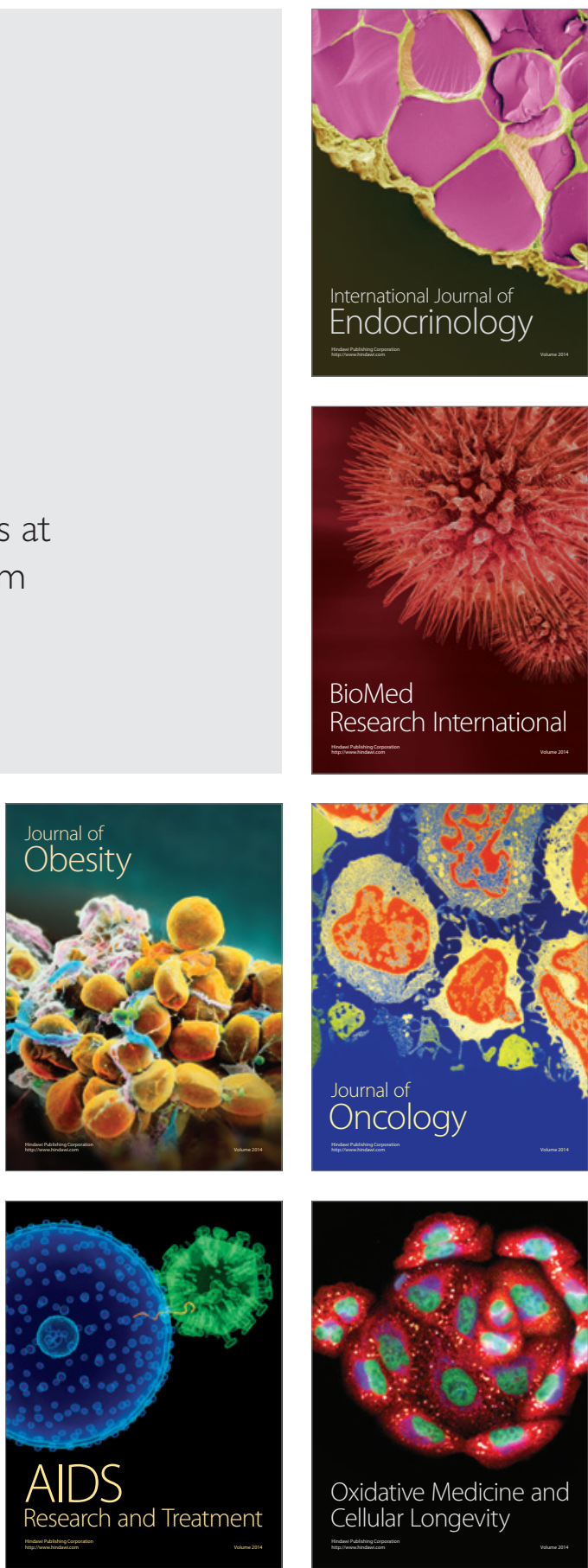